\title{
Vertex transitive maps on the Klein bottle
}

\author{
Ondrej Šuch * \\ Institute of Mathematics and Computer Science \\ Ďumbierska 1, 97401 Banská Bystrica, Slovak Republic \\ and \\ Fakulta prírodných vied, Univerzita Mateja Bela \\ Tajovského 40, 97401 Banská Bystrica, Slovak Republic
}

Received 10 December 2009, accepted 4 August 2011, published online 10 October 2011

\begin{abstract}
We examine properties of groups associated with vertex transitive maps on the Klein bottle. As an application, we prove that all 4-line vertex transitive maps on the Klein bottle introduced by Babai admit a free vertex transitive action, but there are others that do not.

Keywords: Klein bottle, wallpaper group, vertex-transitive map.

Math. Subj. Class.: 57M60, 05C10; $20 \mathrm{H15}$
\end{abstract}

\section{Introduction}

This paper is motivated by a question raised in [8], namely whether all vertex transitive maps on the Klein bottle admit a free vertex-transitive action as is the case on torus. Vertex transitive maps on the Klein bottle and groups acting on them have been considered in the works of Babai [1] and Thomassen [9]. In this work we take a different approach, working exclusively on the universal covering space of the Klein bottle, the plane.

Suppose a map $M$ on the Klein bottle $K B$ is given with a vertex-transitive action, by a group $G$. The map lifts to the universal covering space [1, p. 610], where one obtains a vertex transitive action $[3,4,5]$ of a wallpaper group [12] $\Gamma$ on a semiregular tiling $[6,11]$ $\mathcal{T}$, together with a normal subgroup $K$ (isomorphic to $p g$ ) of transformations preserving the canonical map $\mathbf{R}^{2} \rightarrow K B$. One can recover $G$ as the quotient $\Gamma / K$. The action of $G$ is free if and only if the action of $\Gamma$ is free.

In this paper we aim to provide an insight into the groups involved (Proposition 2.1, Table 1 and 2) and resolve the question of free action (Proposition 5.1).

${ }^{*}$ Research on this work was supported by VEGA grant 1/0722/08.

E-mail address: ondrejs@ @avbb.sk (Ondrej Šuch) 


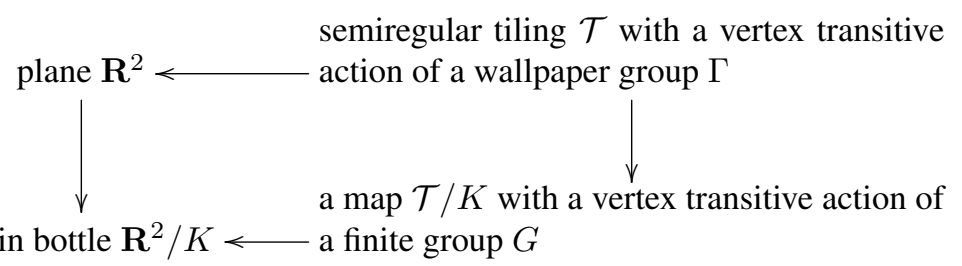

\section{KBU groups}

The group $p g$ is central in our work. It can be introduced in several equivalent ways.

- geometrically by giving two parallel glides $P, Q$ of equal length,

- geometrically by giving a glide $g$ and an orthogonal translation $t$,

- as an abstract group $\left\langle P, Q ; P^{2}=Q^{2}\right\rangle$,

- or as an abstract group $\left\langle g, t ; g t g^{-1}=t^{-1}\right\rangle$.

By a wallpaper or plane crystallographic group one means a group having a discrete action on the plane, and containing two linearly independent translations. There are 17 isomorphism types of wallpaper groups [2]. Not every wallpaper group gives rise to an action on the Klein bottle, because some have no glides, and hence no subgroup isomorphic to $p g$. We shall call a $K B U$ group a wallpaper group that contains a normal subgroup isomorphic to $\mathrm{pg}$.

Proposition 2.1. A wallpaper group $\Gamma$ is a KBU group if and only if $\Gamma$ is isomorphic to one of the following groups: $p m, p g, \mathrm{~cm}$, pmm, pmg, pgg.

Proof. Suppose $\Gamma$ is a KBU group. Clearly, $\Gamma$ cannot be $p 1$ or $p 2$, because these groups do not contain any glides. Suppose $\Gamma$ contains a rotation $r$ of degree $>2$. If $g$ is a glide from the normal subgroup $K$ isomorphic to $\mathrm{pg}$, then $g$ fixes a line $L$. Using the elementary Lemma 2.2 we see that $r g r^{-1}$ fixes $r \cdot L$. It follows that $K$ contains non-parallel glides, and hence a rotation. This contradicts the fact that $p g$ is torsion-free.

It remains to show that the group listed have normal subgroups isomorphic to $p g$. Such subgroups for all of them are listed in Table 2.

Lemma 2.2. Suppose group $G$ acts on a set $X$. Suppose a subgroup $H$ leaves a subset $Y$ fixed. If $g$ is any element of $G$ then the subgroup $g \mathrm{Hg}^{-1}$ leaves a subset $g Y$ fixed.

Let us recall presentations of KBU groups from [2]:

$$
\begin{aligned}
& p m:=\left\langle X, Y, R ; X Y=Y X, R Y=Y R,(R X)^{2}=R^{2}=1\right\rangle \\
& p g:=\left\langle P, Q ; P^{2}=Q^{2}\right\rangle \\
& \mathrm{cm}:=\left\langle R, S ;(R S)^{2}=(S R)^{2}, R^{2}=1\right\rangle \\
& p m m:=\left\langle R_{1}, R_{2}, R_{3}, R_{4} ; R_{1}^{2}=R_{2}^{2}=R_{3}^{2}=R_{4}^{2}=1,\right. \\
&\left.\left(R_{1} R_{2}\right)^{2}=\left(R_{2} R_{3}\right)^{2}=\left(R_{3} R_{4}\right)^{2}=\left(R_{4} R_{1}\right)^{2}=1\right\rangle \\
& p m g:=\left\langle R, T_{1}, T_{2} ; R^{2}=T_{1}^{2}=T_{2}^{2}=1, T_{1} R T_{1}=T_{2} R T_{2}\right\rangle \\
& p g g:=\left\langle P, O ;(P O)^{2}=\left(P^{-1} O\right)^{2}=1\right\rangle \\
& \mathrm{cmm}:=\left\langle T, R_{1}, R_{2} ; T^{2}=R_{1}^{2}=R_{2}^{2}=1,\left(R_{1} R_{2}\right)^{2}=\left(R_{1} T R_{2} T\right)^{2}=1\right\rangle
\end{aligned}
$$


Even though the groups are given in terms of presentations, their elements can be classified in geometric terms. Let $g$ be an element of $\Gamma$. If the centralizer $Z(g)$ of $g$ is a free abelian group of rank 2 , then $g$ is a translation. The translations form a free abelian subgroup $T(\Gamma)$ of rank two, of finite index $(\leq 4)$ in $\Gamma$. Suppose next that the intersection $Z(g) \cap T(g)$ is a free abelian group of rank 1. Then if $g$ is an involution, then $g$ is a mirror, otherwise $g$ is a glide (and $g$ is of infinite order). In the remaining cases $g$ is a rotation.

\subsection{Translations and other elements in KBU groups}

Geometric representations of KBU groups can be seen in Figure 1 with indicated generators. Each picture shows the basis of the translation subgroup (the bounding parallelogram), glides (dotted lines), mirrors (thick lines) and rotations by angle $\pi$ (diamonds). We will now summarize the structure of the translation subgroups of the above seven classes of wallpaper groups.

\subsubsection{Elements of $p m$}

The group is generated by three elements: a pair of orthogonal translations $X$ and $Y$, and a mirror in the direction of $Y$. The translation subgroup is generated by $X$ and $Y$ and is of index 2 in $p m$. There is a coset decomposition $p m=T(p m) \cup R \cdot T(p m)$. Elements of form $R X^{k} Y^{l}$ are mirrors and glides according to whether $l=0$ or not. From definining relations it follows that

$$
R X=X^{-1} R \quad R Y=Y R
$$

\subsubsection{Elements of $p g$}

The group is generated by a pair of parallel glides $P$ and $Q$ satisfying $P^{2}=Q^{2}$. The translation subgroup is generated by $P^{2}$ and $P^{-1} Q$ and is of index $2 \mathrm{in} p g$. There is a coset decomposition $p g=T(p g) \cup P \cdot T(p g)$. Elements of form $P \cdot P^{2 l}\left(P^{-1} Q\right)^{k}$ are all glides. We have

$$
P \cdot P^{2}=P^{2} \cdot P \quad P\left(P^{-1} Q\right)=\left(P^{-1} Q\right)^{-1} P
$$

\subsubsection{Elements of $\mathrm{cm}$}

The group is generated by a translation $S$ and a mirror $R$, which are neither parallel, nor orthogonal. The translation subgroup is generated by $S$ and $R S R$ and is of index 2 in $\mathrm{cm}$. There is a coset decomposition $\mathrm{cm}=T(\mathrm{~cm}) \cup R \cdot T(\mathrm{~cm})$. Elements of form $R S^{k}(R S R)^{l}$ are mirrors and glides according to whether $k=-l$ or not. From defining relations it follows

$$
R \cdot S=R S R \cdot R \quad R \cdot R S R=S \cdot R
$$

\subsubsection{Elements of $\mathrm{pmm}$}

This group is generated by four mirrors $R_{1}-R_{4}$ forming the sides of a square. The translation subgroup is generated by $R_{1} R_{3}$ and $R_{2} R_{4}$, and is of index 4 in $\mathrm{pmm}$. There is a coset decomposition $\mathrm{pmm}=T(\mathrm{pmm}) \cup R_{1} \cdot T(\mathrm{pmm}) \cup R_{2} \cdot T(\mathrm{pmm}) \cup R_{1} R_{2} \cdot T(\mathrm{pmm})$. All 


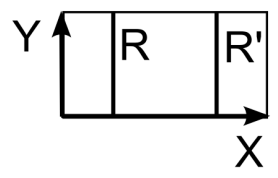

(a) Group pm

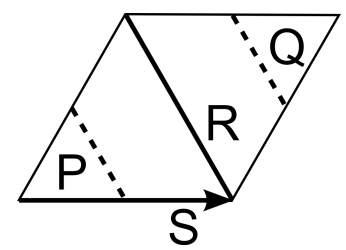

(c) Group $\mathrm{cm}$ (b) Group $p g$

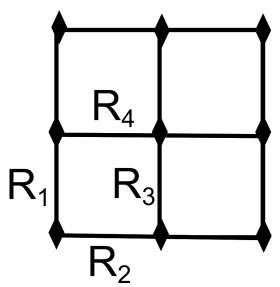

(d) Group pmm
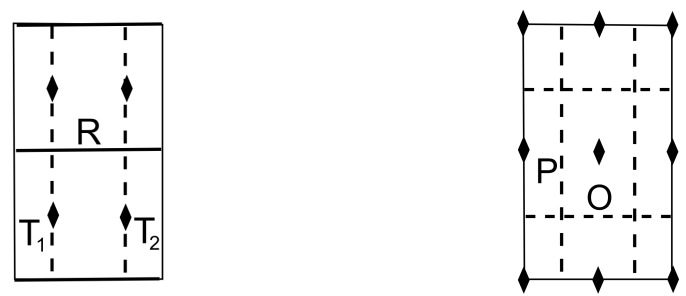

(e) Group pmg

(f) Group pgg

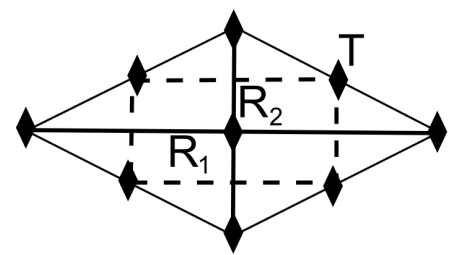

(g) Group cmm

Figure 1: KBU groups. 
elements of the last coset are rotations. Elements of the form $R_{1}\left(R_{1} R_{3}\right)^{k}$ and $R_{2}\left(R_{2} R_{4}\right)^{k}$ are mirrors, and the remaining non-translations are glides. From the defining relations we have:

$$
\begin{aligned}
R_{1}\left(R_{2} R_{4}\right) & =\left(R_{2} R_{4}\right) R_{1} & R_{1}\left(R_{1} R_{3}\right) & =\left(R_{1} R_{3}\right)^{-1} R_{1} \\
R_{2}\left(R_{2} R_{4}\right) & =\left(R_{2} R_{4}\right)^{-1} R_{2} & R_{2}\left(R_{1} R_{3}\right) & =\left(R_{1} R_{3}\right) R_{2} \\
\left(R_{1} R_{2}\right)\left(R_{2} R_{4}\right) & =\left(R_{2} R_{4}\right)^{-1}\left(R_{1} R_{2}\right) & \left(R_{1} R_{2}\right)\left(R_{1} R_{3}\right) & =\left(R_{1} R_{3}\right)^{-1}\left(R_{1} R_{2}\right)
\end{aligned}
$$

\subsubsection{Elements of $\mathrm{pmg}$}

This group is generated by a mirror $R$ and two translations $T_{1}, T_{2}$ lying on a line parallel to it. The translation subgroup is generated by $\left(T_{1} R\right)^{2}$ and $T_{1} T_{2}$, and is of index 4 in $p m g$. There is a coset decomposition $p m m=T(p m g) \cup R \cdot T(p m g) \cup T_{1} \cdot T(p m g) \cup R T_{1}$. $T(p m g)$. We have

$$
\begin{aligned}
R\left(T_{1} T_{2}\right) & =\left(T_{1} T_{2}\right) R & \left(T_{1} R\right)^{2} \cdot R & =R \cdot\left(T_{1} R\right)^{-2} \\
T_{1}\left(T_{1} T_{2}\right) & =\left(T_{1} T_{2}\right)^{-1} T_{1} & T_{1}\left(T_{1} R\right)^{2} & =\left(T_{1} R\right)^{-2} T_{1} \\
\left(R T_{1}\right)\left(T_{1} T_{2}\right) & =\left(T_{1} T_{2}\right)^{-1}\left(R T_{1}\right) & \left(R T_{1}\right)\left(T_{1} R\right)^{2} & =\left(T_{1} R\right)^{2}\left(R T_{1}\right)
\end{aligned}
$$

\subsubsection{Elements of $p g g$}

This group is generated by a pair of orthogonal glides $O$ and $P$. The translation subgroup is generated by $P^{2}$ and $O^{2}$. There is a coset decomposition pgg $=T(p g g) \cup O \cdot T(p g g) \cup$ $P \cdot T(p g g) \cup(O P) \cdot T(p g g)$. We have

$$
\begin{array}{rlrl}
P \cdot P^{2} & =P^{2} \cdot P & P \cdot O^{2} & =O^{-2} \cdot P \\
O \cdot P^{2} & =P^{-2} \cdot O & O \cdot O^{2} & =O^{2} \cdot O \\
O P \cdot P^{2} & =P^{-2} \cdot O P & O P \cdot O^{2} & =O^{-2} \cdot O P
\end{array}
$$

\subsubsection{Elements of $\mathrm{cmm}$}

This group is generated by a pair of orthogonal mirrors $R_{1}, R_{2}$ and a rotation not lying on any of them. The translation subgroup is generated by $R_{1} R_{2} T$ and $R_{2} T R_{1}$. We have a coset decomposition $\mathrm{cmm}=T(\mathrm{cmm}) \cup R_{1} \cdot T(\mathrm{cmm}) \cup R_{2} \cdot T(\mathrm{cmm}) \cup T \cdot T(\mathrm{cmm})$. We have

$$
\begin{array}{rlrl}
R_{1}\left(R_{1} R_{2} T\right) & =\left(R_{2} T R_{1}\right) R_{1} & R_{1}\left(R_{2} T R_{1}\right) & =\left(R_{1} R_{2} T\right) R_{1} \\
R_{2}\left(R_{1} R_{2} T\right) & =\left(R_{2} T R_{1}\right)^{-1} R_{2} & R_{2}\left(R_{2} T R_{1}\right) & =\left(R_{1} R_{2} T\right)^{-1} R_{2} \\
T\left(R_{1} R_{2} T\right) T & =\left(R_{1} R_{2} T\right)^{-1} T & T\left(R_{2} T R_{1}\right) & =\left(R_{2} T R_{1}\right)^{-1} T
\end{array}
$$

\section{Ten families of glides of $\mathrm{KBU}$ groups}

In order to analyze normal subgroups of KBU groups isomorphic with $p g$ it is first necessary to understand the set of glides in these wallpaper groups.

In Table 1 we list ten families of glides parametrized by positive integers $n>0$. Using the facts from the previous section it is straightforward to verify that the elements $g$ 
listed there are glides, that is they are not involutions, and centralize a rank 1 subgroup of translations. The subgroup of translations is in fact generated by the corresponding parallel translation.

\begin{tabular}{|l|c|c|c|c|}
\hline Family & Group $G$ & glide $g$ & orthogonal translation & parallel translation \\
\hline M1 & $p m$ & $R Y^{n}$ & $X$ & $Y$ \\
M2 & $p m m$ & $R_{1}\left(R_{2} R_{4}\right)^{n}$ & $R_{1} R_{3}$ & $R_{2} R_{4}$ \\
M3 & $p m g$ & $R\left(T_{1} T_{2}\right)^{n}$ & $\left(T_{1} R\right)^{2}$ & $T_{1} T_{2}$ \\
M4 & $c m$ & $R(R S)^{2 n}$ & $R S R S^{-1}$ & $R S R S$ \\
M5 & $c m m$ & $R_{1}\left(T R_{2}\right)^{2 n}$ & $\left(T R_{1}\right)^{2}$ & $\left(T R_{2}\right)^{2}$ \\
\hline G1 & $p g$ & $P^{2 n-1}$ & $\left(P Q^{-1}\right)^{2}$ & $P^{2}$ \\
G2 & $p m g$ & $\left(T_{1} R\right)^{2 n-1}$ & $T_{1} T_{2}$ & $\left(T_{1} R\right)^{2}$ \\
G3 & $p g g$ & $O^{2 n-1}$ & $P^{2}$ & $O^{2}$ \\
G4 & $c m$ & $(R S)^{2 n-1}$ & $R S R S^{-1}$ & $R S R S$ \\
G5 & $c m m$ & $\left(R_{1} T\right)^{2 n-1}$ & $\left(R_{2} T\right)^{2}$ & $\left(R_{1} T\right)^{2}$ \\
\hline
\end{tabular}

Table 1: Glides in KBU groups.

Take for instance the glide $g=\left(T_{1} R\right)^{2 n-1}$ in family G2. The translation subgroup of pmg is generated by $\left(T_{1} R\right)^{2}$ and $T_{1} T_{2}$. It is clear that $g$ commutes with $\left(T_{1} R\right)^{2}$, and using equations from 2.1 .5 we check that it anticommutes with $T_{1} T_{2}$ :

$$
\begin{aligned}
g\left(T_{1} T_{2}\right) g^{-1} & =\left(T_{1} R\right)^{2 n-1}\left(T_{1} T_{2}\right)\left(R T_{1}\right)^{2 n-1} \\
& =\left(T_{1} R\right)^{2 n-1}\left(R T_{1}\right)^{2 n-1}\left(T_{1} T_{2}\right)^{(-1)^{2 n-1}} \\
& =\left(T_{1} T_{2}\right)^{-1}
\end{aligned}
$$

Since $g^{2}=\left(\left(T_{1} R\right)^{2}\right)^{2 n-1}$ we see that $g$ is not an involution, and thus is not a mirror.

The list provided in Table 1 is in a way exhaustive.

Proposition 3.1. If $\gamma$ is a glide in $K B U$ group $\Gamma$, then there exists $n \geq 1$ and an isomorphism $\Gamma \rightarrow G$, such that $\gamma$ maps to one of the glides $g$ listed in Table 1.

Proof. Suppose $\gamma$ is a glide. Clearly, $\gamma$ cannot belong to $T(\Gamma)$. If $\gamma$ belonged to $\alpha \cdot T(\Gamma)$ for some rotation $\alpha$, then $\gamma^{2}=1$, which contradicts the fact that all glides are of infinite order. Let us now distinguish several cases.

Suppose next $\Gamma$ admits an orthogonal basis of $T(\Gamma)$. This is true for groups $p m, p g$, pmm, pmg, pgg. As shown in 2.1 we need to distinguish two cases.

Firstly suppose $\gamma=R X^{k} Y^{l}$, where $X$ and $Y$ is an orthogonal basis of $T(\Gamma)$ such that

$$
R X=X^{-1} R \quad R Y=Y R
$$

Then $\gamma^{2}=Y^{2 l}$ and thus $\gamma$ is a glide if and only if $l \neq 0$. The next step is to show that there exists an automorphism of $\Gamma$ such that $R Y^{l}$ is mapped to $R Y^{-l}$. In the case of groups $p m m, p m g, p g g$ we can take it to be conjugation by any rotation. For groups $p m$ and $p g$, any rotation of plane of order 2 accomplishes the same, but in these cases it is an outer automorphism. Conjugating $R Y^{l}$ by $X^{s}$ we obtain

$$
X^{s} R X^{k} Y^{l} X^{-s}=R X^{k-2 s} Y^{l} \quad X^{-s} R X^{k} Y^{l} X^{s}=R X^{k+2 s} Y^{l}
$$


Thus modulo inner automorphisms there are at most two classes of glides in the form $R X^{k} Y^{l}$. These are exchanged by conjugation with $\frac{1}{2} X$, which is an outer automorphism. In the case of group pmm we remark that the map

$$
\phi\left(R_{1}\right):=R_{2}, \quad \phi\left(R_{2}\right):=R_{3}, \quad \phi\left(R_{3}\right):=R_{4}, \quad \phi\left(R_{4}\right):=R_{1}
$$

is an outer automorphism exchanging glides $R_{1}\left(R_{2} R_{4}\right)^{k}$ with $R_{2}\left(R_{3} R_{1}\right)^{k}$.

Secondly, we need to consider the case $\gamma=P \cdot P^{2 k} Y^{l}$, where $P$ is a glide and $Y$ is an orthogonal translation satisfying

$$
P Y=Y^{-1} P
$$

Then $\gamma^{2}=P^{4 k+2}$, which shows that $\gamma$ is a glide for all choices of $k$ and $l$. Arguing as in the previous case we obtain that modulo automorphisms of the group two glides $P \cdot P^{2 k} Y^{l}$ and $P \cdot P^{2 k^{\prime}} Y^{l^{\prime}}$ are equivalent if and only if $k= \pm k^{\prime}$.

Suppose now that $\Gamma$ is isomorphic to $\mathrm{cm}$ or $\mathrm{cmm}$. We can assume that the $\gamma$ belongs to a coset $R T(\Gamma)$, where $R$ is a mirror and $T(\Gamma)$ is generated by (equal length) translations $S, R S R$. Then we can explicitly write

$$
\begin{aligned}
\gamma & =R S^{k}(R S R)^{l} \\
\gamma^{2} & =S^{k+l}(R S R)^{k+l}
\end{aligned}
$$

and thus $\gamma$ is a glide if and only if $k+l \neq 0$. In case of $\mathrm{cmm}$ any rotation in $\Gamma$ conjugates $R S^{k}(R S R)^{l}$ with $R S^{-k}(R S R)^{-l}$. In case of $\mathrm{cm}$ any plane rotation does the same, but it is now an outer automorphism. Conjugating by $S^{s}$ gives

$$
S^{s}\left(R S^{k}(R S R)^{l}\right) S^{-s}=R S^{k-s}(R S R)^{l+s} .
$$

This shows that if $k+l=2 n$, then the glide $R S^{k}(R S R)^{l}$ is conjugate with $R S^{n}(R S R)^{n}=$ $R(S R)^{2 n}=R(R S)^{2 n}$. If on the other hand $k+l=2 n+1$ the the glide $R S^{k}(R S R)^{l}$ is conjugate with $R S S^{n}(R S R)^{n}=R S(R S R)^{n} S^{n}=(R S)^{2 n+1}$.

\section{Groups acting on the Klein bottle}

In the previous section we listed ten families of glides in KBU groups. We now consider the problem of determining the normal closure of a glide $g$ in a KBU group. Conjugating by an orthogonal translation in view of Lemma 2.2 gives a different, parallel glide $g^{\prime}$. Thus the normal closure always contains a subgroup isomorphic to $\mathrm{pg}$.

On the other hand, any KBU group is a subgroup of finite index in pmm ([2]). This can also be seen geometrically, or by giving explicit injections:

$$
\begin{aligned}
i_{1}: p m \rightarrow p m m & i_{1}(X):=R_{1} R_{3}, i_{1}(Y):=R_{2} R_{4}, i_{1}(R):=R_{1} \\
i_{2}: p g \rightarrow p m m & i_{2}(P):=R_{1} R_{2} R_{4}, i_{2}(Q):=R_{1} R_{3} R_{1} R_{2} R_{4} \\
i_{3}: \mathrm{cm} \rightarrow p m m & i_{3}(R):=R_{1}, i_{3}(S):=\left(R_{1} R_{3}\right)\left(R_{2} R_{4}\right) \\
i_{4}: p m g \rightarrow p m m & i_{4}(P):=R_{1} R_{2} R_{4}, i_{4}(Q):=R_{1}\left(R_{1} R_{3}\right)^{2} R_{2} R_{4}, i_{4}(R)=R_{3} \\
i_{5}: p g g \rightarrow p m m & i_{5}(P):=R_{1} R_{2} R_{4}, i_{5}(O):=R_{2} R_{1} R_{3} \\
i_{6}: \mathrm{cmm} \rightarrow p m m & i_{6}\left(R_{1}\right):=R_{1}, i_{6}\left(R_{2}\right):=R_{2}, i_{6}(T):=R_{3} R_{4}
\end{aligned}
$$


The normal closure of a glide in a KBU group will thus be a subgroup of the normal closure of a glide in $\mathrm{pmm}$. Without loss of generality, consider the normal closure of the glide $g=R_{1}\left(R_{2} R_{4}\right)^{n}$ in $p m m$. We have

$$
\begin{aligned}
& R_{1} g R_{1}=g \\
& R_{2} g R_{2}=R_{2} R_{1}\left(R_{2} R_{4}\right)^{n} R_{2}=R_{1}\left(R_{4} R_{2}\right)^{n}=g^{-1} \\
& R_{3} g R_{3}=R_{3} R_{1}\left(R_{2} R_{4}\right)^{n} R_{3}=R_{3} R_{1} R_{3}\left(R_{2} R_{4}\right)^{n}=\left(R_{3} R_{1}\right)^{2} g \\
& R_{4} g R_{4}=R_{4} R_{1}\left(R_{2} R_{4}\right)^{n} R_{4}=R_{1}\left(R_{4} R_{2}\right)^{n}=g^{-1}
\end{aligned}
$$

One readily checks that $R_{2}$ and $R_{4}$ fix the translation $\left(R_{3} R_{1}\right)^{2}$, while $R_{1}$ and $R_{3}$ invert it. It follows that the normal closure of $g$ in $\mathrm{pmm}$ is the subgroup isomorphic to $\mathrm{pg}$ generated by $g$ and the translation $\left(R_{3} R_{1}\right)^{2}$ orthogonal to $g$. We have arrived at the following lemma.

Lemma 4.1. The normal closure of a glide in a KBU group is a subgroup isomorphic to $p g$.

Proof. Indeed, the normal closure contains two parallel glides, and since it is a subgroup of $p g$, which contains no mirrors, nor rotations, it has to be isomorphic to $p g$.

By geometric analysis using Lemma 2.2 one can determine the normal closure of glides in KBU groups precisely.

Proposition 4.2. The normal closure of a glide $g$ in a KBU group $G$ for families M1-M5, G1-G5 is the group $\langle g, t\rangle$ as given in Table 2. One has $\mathrm{gtg}^{-1}=t^{-1}$, with the indicated quotient and a set of generators of $G /\langle g, t\rangle$.

\begin{tabular}{|l|c|c|c|c|c|}
\hline Family & Group $G$ & glide $g$ & translation $t$ & $F:=G /\langle g, t\rangle$ & Generators of $F$ \\
\hline M1 & $p m$ & $R Y^{n}$ & $X^{2}$ & $\mathbf{Z}_{2} \times \mathbf{Z}_{2 n}$ & $\bar{X} ; \bar{Y}$ \\
M2 & $p m m$ & $R_{1}\left(R_{2} R_{4}\right)^{n}$ & $\left(R_{1} R_{3}\right)^{2}$ & $\mathbf{Z}_{2} \times D_{4 n}$ & $\bar{R}_{1} \bar{R}_{3} ; \bar{R}_{2}, \bar{R}_{4}$ \\
M3 & $p m g$ & $R\left(T_{1} T_{2}\right)^{n}$ & $\left(T_{1} R\right)^{2}$ & $D_{4 n}$ & $\bar{T}_{1} \bar{T}_{2}, \bar{T}_{1}$ \\
M4 & $c m$ & $R(R S)^{2 n}$ & $R S R S^{-1}$ & $\mathbf{Z}_{4 n}$ & $\bar{R} \bar{S}$ \\
M5 & $c m m$ & $R_{1}\left(T R_{2}\right)^{2 n}$ & $\left(T R_{1}\right)^{2}$ & $D_{8 n}$ & $\bar{T} \bar{R}_{2}, \bar{R}_{2}$ \\
\hline G1 & $p g$ & $P^{2 n-1}$ & $\left(P Q^{-1}\right)^{2}$ & $\mathbf{Z}_{2(2 n-1)}$ & $\bar{Q}$ \\
G2 & $p m g$ & $\left(T_{1} R\right)^{2 n-1}$ & $\left(T_{1} T_{2}\right)^{2}$ & $\mathbf{Z}_{2} \times D_{2(2 n-1)}$ & $\bar{T}_{1} \bar{T}_{2} ; \bar{T}_{1} R, \bar{R}$ \\
G3 & $p g g$ & $O^{2 n-1}$ & $P^{2}$ & $D_{2(2 n-1)}$ & $\bar{O}, \bar{P}$ \\
G4 & $c m$ & $(R S)^{2 n-1}$ & $R S R S^{-1}$ & $\mathbf{Z}_{2(2 n-1)}$ & $\bar{S}$ \\
G5 & $c m m$ & $\left(R_{1} T\right)^{2 n-1}$ & $\left(R_{2} T\right)^{2}$ & $\mathbf{Z}_{2} \times D_{2(2 n-1)}$ & $\bar{R}_{2} ; \bar{R}_{1} \bar{T}_{1}, \bar{R}_{1}$ \\
\hline
\end{tabular}

Table 2: Group factors of KBU groups.

Proof. Determination of normal closure in each case proceeds analogously to the case of M2 analyzed above.

Let us illustrate how one proves the rest in the case G5. First, since

$$
R_{2} T R_{1} \cdot R_{1} R_{2} T=\left(R_{2} T\right)^{2}
$$


we have from (2.1) and (2.3)

$$
\begin{aligned}
g t g^{-1} & =\left(R_{1} T\right)^{2 n-1}\left(R_{2} T\right)^{2}\left(T R_{1}\right)^{2 n-1}=\left(R_{2} T\right)^{-2}\left(R_{1} T\right)^{2 n-1}\left(T R_{1}\right)^{2 n-1} \\
& =\left(R_{2} T\right)^{-2}=t^{-1}
\end{aligned}
$$

Since $R_{1} \cdot R_{1} T=T$, the images $\bar{R}_{1} \bar{T}, \bar{R}_{1}, \bar{R}_{2}$ of elements $R_{1} T, R_{1}, R_{2}$ generate $F$. Elements $R, T_{1}, T_{2}$ are all involutions. From $\left(R_{1} R_{2}\right)^{2}=1$ it follows that $R_{2}$ commutes with $R_{1}$, and from $\left(\bar{R}_{2} \bar{T}\right)^{2}=1$ it follows that $\bar{R}_{2}$ commutes with $\bar{T}$. We can now compute the quotient (omitting redundant relations):

$$
\begin{aligned}
c m m /\langle g, t\rangle & =\left\langle\bar{T}, \bar{R}_{1}, \bar{R}_{2} ; \bar{T}^{2}=\bar{R}_{1}=\bar{R}_{2}^{2}=1,\left(\bar{R}_{1} \bar{T}\right)^{2 n-1}=\left[\bar{R}_{2}, \bar{T}\right]=\left[\bar{R}_{2}, \bar{R}_{1}\right]=1\right\rangle \\
& =\left\langle\bar{R}_{2} ; \bar{R}_{2}^{2}=1\right\rangle \times\left\langle\bar{R}_{1}, \bar{T} ; \bar{R}_{1}^{2}=\bar{T}^{2}=\left(\bar{R}_{1} \bar{T}\right)^{2 n-1}=1\right\rangle \\
& =\mathbf{Z}_{2} \times D_{2(2 n-1)}
\end{aligned}
$$

Corollary 4.3. (Babai) A group $G$ acts on the Klein bottle if and only if it is a subgroup of $\mathbf{Z}_{2} \times D_{2 n}$ for some $n \geq 1$.

Proof. It is known ([1] Lemma 7.3 and [7] Theorem 6.2.4, also [10]) that if a group $H$ acts on a surface, then there exists a Cayley map for this group on the surface. Thus any group action can be lifted to a Cayley action of a KBU group $\Gamma$ on a semiregular tiling, such that it has a normal subgroup $K$ isomorphic to $\mathrm{pg}$ that preserves the canonical projection. One has $H=\Gamma / K$. The group $K$ contains a glide $\gamma$, and since it is normal, it contains the normal closure of $\gamma$. From the previous proposition it follows that $H$ is isomorphic to a factor of $F$ from Table 1, all of which are subgroups of $\mathbf{Z}_{2} \times D_{2 n}$.

Conversely, groups $\mathbf{Z}_{2} \times D_{2 n}$ act on the Klein bottle for both $n$ even ( $p m m$ with glide family M2), and $n$ odd ( $p m g$ with glide family G2 or $\mathrm{cmm}$ with glide family G5).

\section{Vertex-transitive maps on the Klein bottle}

In Babai's work [1] one can find a list of vertex-transitive maps on the Klein bottle. Let us explain his terminology. He represents semiregular tilings with letters as follows:
A the triangle tiling
B the square tiling
$\mathrm{C}$ the hexagonal tiling
$\mathrm{D}$ the elongated square tiling
E the snub square tiling
$\mathrm{F}$ the truncated square tiling

Then he shows 12 families of positions for glides acting on the tilings A1, A2, B1, B2, C1C3, D1, D2, E1, F1, F2. Each of them has the property that there is a glide $g$ in the position, and a translation $t$ orthogonal to $g$ such that the quotient of the plane by the group $\langle g, t\rangle$ is a vertex-transitive map on the Klein bottle. Moreover, the vertices of the semiregular tiling fall into 4 lines modulo translations by $t$. He calls the last property a 4-line condition. He notes that every position, except B1, has a natural parity condition on the glides in the 


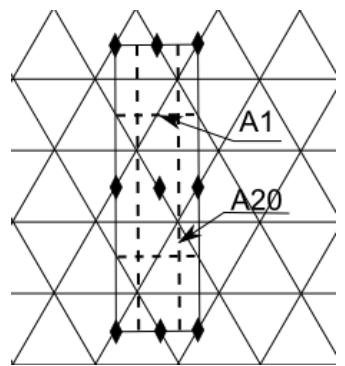

(a) pgg action on the triangular tiling

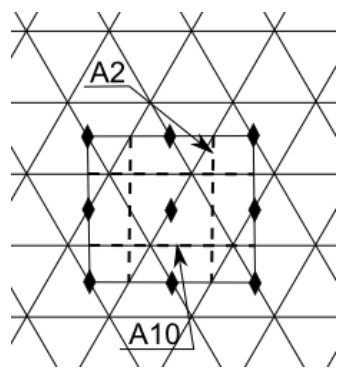

(d) pgg action on the triangular tiling

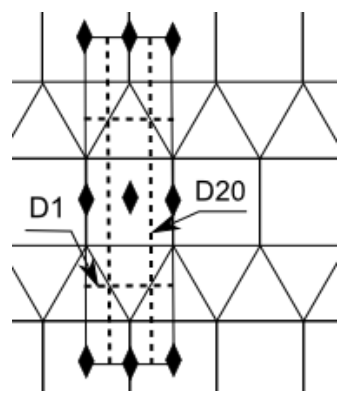

(g) pgg action on the elongated triangular tiling

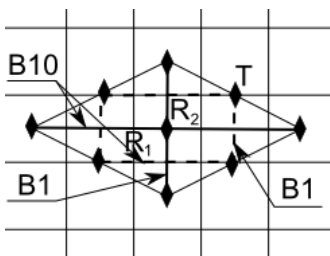

(b) $\mathrm{cmm}$ action on the square tiling
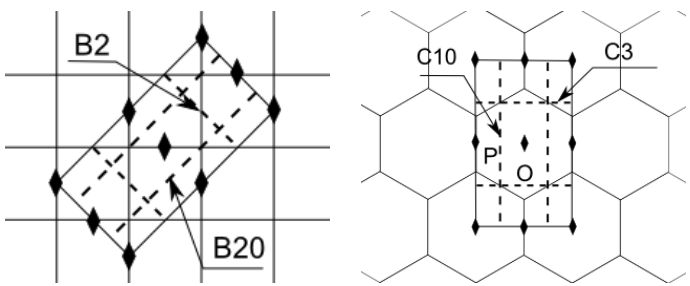

(e) $p g g$
tiling

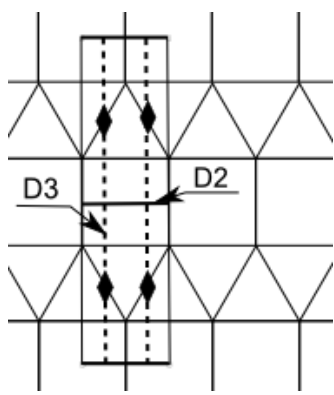

nal tiling

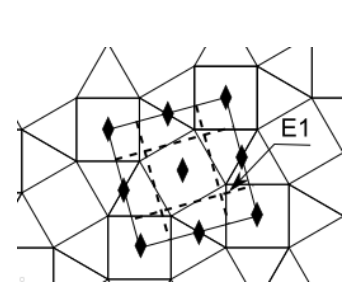

(h) pmg action on the elon-

(i) pgg action on the snub square tiling

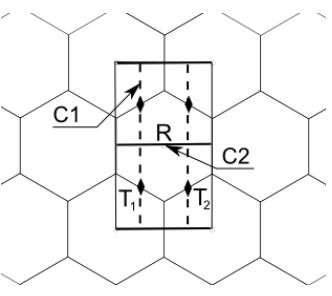

(c) pmg action on the hexagonal tiling

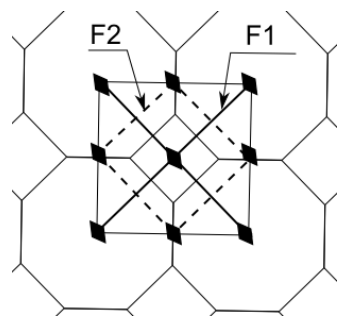

(j) $\mathrm{cmm}$ action on the truncated square tiling 
position. This results in 13 families of 4-line vertex-transitive maps, and he lists 5 2-line families (A10, A20, B10, B20, D10) and one 1-line family arising from the square tiling.

We can now state our final result.

Proposition 5.1. All 4-line vertex transitive maps on the Klein bottle admit a free vertex transitive action. There are however vertex-transitive maps on the Klein bottle that do not admit a free vertex transitive action.

Proof. The first statement is demonstrated by exhibiting free vertex transitive actions for each of Babai's families. This is done in Figures 2(a)-2(j). Note that for position B1, we show a free vertex transitive action for both even and odd glides [1]. Also note that for positions C1, D3, the resulting maps are indeed 4-line. This is because in the family G2, the normal closure is generated by the square of the smallest translation orthogonal to the glide.

We will prove the second statement by contradiction. Let us consider the vertex transitive action of the group $\Gamma=\mathrm{cm}$ on the triangular tiling as shown in Figure 2(k). For any integer $n \geq 1$ one obtains a vertex transitive map on the Klein bottle by taking the quotient by the normal closure in $\Gamma$ of a glide in the direction $g$ of length $n \times$ (length of triangle side). In fact, the images of translations act transitively. This shows there are vertex transitive maps arising from triangle tilings with number of vertices divisible by arbitrarily large power of 2.

Since any mirror of a triangular tiling fixes a vertex of the tiling, there are free vertex transitive actions only by groups $p g$ and $p g g$ on the tiling. Any maps arising from those actions are factors by odd multiple sized glides. Since there are only finitely many classes of actions of $p g$ and $p g g$ modulo automorphisms of the triangle tiling, we conclude that the power of 2 dividing the size of free vertex transitive maps has an upper bound.

This contradiction shows that there are maps that do not admit a free vertex transitive action.

Finally we remark there is a 2-line family of vertex transitive maps for position C10 shown in Figure 2(f), omitted in Babai's list.

\section{References}

[1] L. Babai, Vertex-transitive graphs and vertex-transitive maps, J. Graph Theory 15 (1991), 587 627.

[2] H. S. M. Coxeter and W. O. J. Moser, Generators and Relations for Discrete Groups, 4th ed., 1980, Springer-Verlag, Berlin and New York.

[3] B. N. Delone, Theory of planigons (in Russian), Izv. Akad. Nauk SSSR Ser. Mat. 23 (1959), 365-386.

[4] B. N. Delone and N. P. Dolbilin, M. I. Štogrin, Combinatorial and metric theory of planigons (in Russian), Algebra, number theory and their applications, Trudy Mat. Inst. Steklov. 148 (1978), 109-140, 275.

[5] B. Grünbaum and G. C. Shephard, The ninety-one types of isogonal tilings in the plane, Trans. Amer. Math. Soc. 242 (1978), 335-353.

[6] B. Grünbaum and G. C. Shephard, Tilings and Patterns, W. H. Freeman and Company, 1987.

[7] J. L. Gross and T. W. Tucker, Topological graph theory, Dover, Toronto, 2001. 
[8] O. Šuch, Vertex-transitive maps on a torus, Acta Mathematica Universitatis Comenianae 53 (2011), 1-30.

[9] C. Thomassen, Tilings of the torus and the Klein bottle and vertex-transitive graphs on a fixed surface, Trans. Amer. Math. Soc. 323 (1991), 605-635.

[10] T. W. Tucker, Finite groups acting on surfaces and the genus of a group, J.Combin. Theory Ser. B 34 (1983), 82-98.

[11] http://en.wikipedia.org/wiki/Tilings_of_regular_polygons

[12] http://en.wikipedia.org/wiki/Wallpaper_group 